\title{
Use of computerized tomography of the abdominal wall in the diagnosis of partial post-operative wound dehiscence
}

\author{
James Smith-Behn ${ }^{1}$, Malcolm Arnold ${ }^{1}$ and James Might ${ }^{2}$ \\ Departments of ${ }^{1}$ Surgery and ${ }^{2}$ Internal Medicine, Northeastern Ohio Universities College of Medicine, Youngstown \\ Hospital Assn., Youngstown, Ohio 44503, USA.
}

\begin{abstract}
Summary: A patient had occult post-operative partial wound dehiscence which was accurately diagnosed by performing a CT scan of the abdomen. It is suggested that CT scan of the abdominal wall is useful for early diagnosis of occult abdominal wound dehiscence.
\end{abstract}

\section{Introduction}

Wound dehiscence is an uncommon but troublesome complication of abdominal surgery. While many cases present in a readily recognizable way, some are clinically inapparent, especially in the early stages. We present a case of occult, incomplete abdominal wound dehiscence accurately diagnosed with the aid of the computerized tomographic scanner.

\section{Case report}

A 68 year old markedly obese male who suffered from thigh and leg claudication was referred for revascularization of both lower extremities after angiography revealed complete obstruction of the right common iliac artery and severe stenosis of the proximal left common iliac artery. An aorto-bifemoral graft was placed which, due to the patient's obesity and previous operations in the area required more than the usual time to perform. The early post-operative period was entirely uneventful until the seventh post-operative day when he experienced some pain in the supraumbilical portion of his incision following a paroxysmal sneeze. At this time there was no discharge from the wound, and no cough impulse palpable in this area. A small superficial gap was palpable in the incision about half way between the xiphoid cartilage and the umbilicus. It was not clear from the examination whether this represented merely limited dehiscence of a small portion of the subcutaneous closure or whether a more complete occult dehiscence of the linea alba was present in addition. A computerized tomographic (CT) scan of the abdomen was performed which showed good fascial approximation in the infraum-

Correspondence: J. Smith-Behn, M.D.

Accepted: 17 April 1986 bilical portion, but separation in the supraumbilical portion with haematoma occupying the space between the separated edges of the linea alba (Figure 1). The patient was returned to the operating room where, under local anaesthesia, a one inch segment of the supraumbilical portion of the incision was opened. By palpation it was determined that complete separation of the linea alba repair had occurred between the xyphoid and the umbilicus. The patient was anaesthetized and the incision was explored further. The operative appearances were found to correlate perfectly with the CT scan evidence and in particular the peritoneum was found to be intact. The incision was again closed. The patient made an uneventful recovery and is doing well 18 months post-operatively. There is no clinical evidence of incisional hernia at this point. A repeat CT scan 2 months after discharge is shown in Figure 2.

\section{Discussion}

Computerized tomography of the chest has been used extensively for the diagnosis of intrathoracic pathology as summarized by Webb (1983) and Strickland (1984). Leitman et al. (1983) have proved its efficacy in demonstrating chest wall pathology. Less than obvious post-operative median sternotomy dehiscence and infection are also adequately diagnosed by CT as shown by Harjula \& Jarvinen (1983) and Kay et al. (1983). Rubenstein et al. (1983) have shown its use in the diagnosis of intra-abdominal pathology, and Fisch \& Brodey (1981) have proved its accuracy in the diagnosis of abdominal wall infection, haemorrhage and neoplasm. CT was performed in this patient arising from one of the author's previous observations during CT study of the sternum, that some of the

The Fellowship of Postgraduate Medicine, 1986 


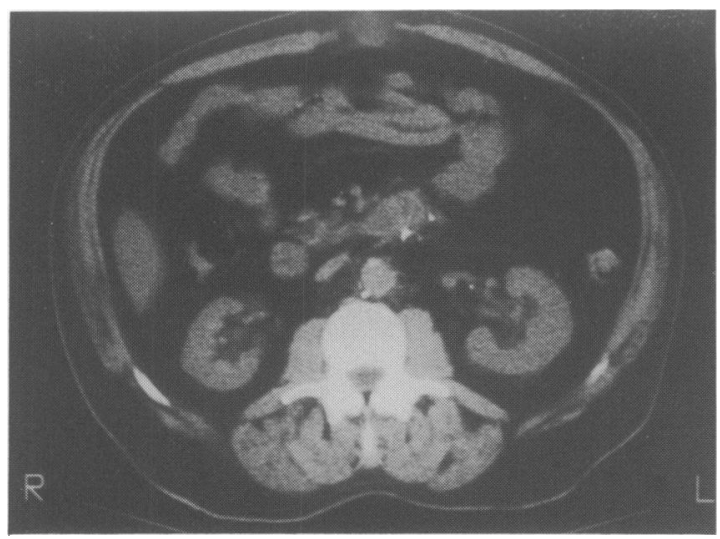

Figure 1 Computerized tomography of the abdomen showing complete separation of the linea alba.

lowest tomographic plates showed very detailed anatomy of the anterior abdominal wall. The skin, subcutaneous tissue, linea alba, anterior and posterior fascial sheaths of the rectus abdominis muscles, the intervening muscle itself, the extra-peritoneal fat and the peritoneum are all well delineated.

When post-operative dehiscence is complete and readily recognizable, clearly, special investigations are unnecessary. The early diagnosis of clinically inapparent incomplete dehiscence is of more than

\section{References}

FISCH, A. \& BRODEY, P. (1981). Computed tomography of the anterior abdominal wall: normal anatomy and pathology. Journal of Computer Assisted Tomography, 5, 728.

HARJULA, A. \& JARVINEN, A. (1983). Postoperative median sternotomy dehiscence. Scandinavian Journal of Thoracic and Cardiovascular Surgery, 17, 277.

KAY, H., GOODMAN, L., TEPLIC, S. \& MUNDTH, E. (1983). Use of computed tomography to assess mediastinal complications after median sternotomy. Annals of Thoracic Surgery, 36, 706.

LEITMAN, B., MCCAULEY, D., REEDE, D., FIROOZNIA, H.,

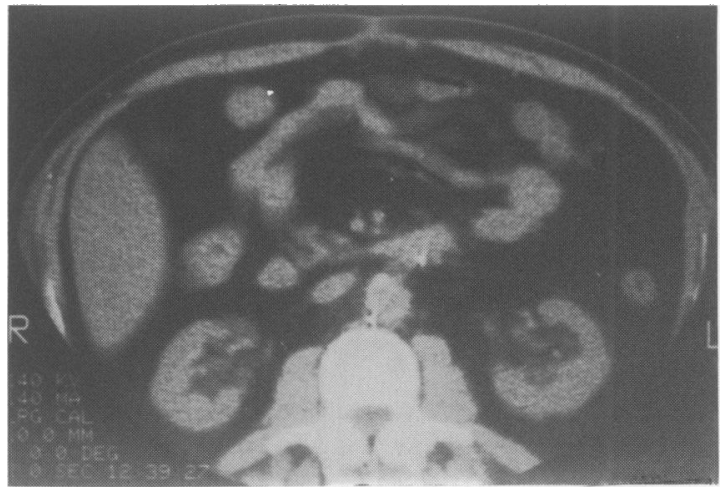

Figure 2 Computerized tomography of the abdomen post-operatively showing re-approximation of the linea alba.

academic importance. With early recognition and surgical revision the uncommon late dehiscence following hospital discharge and, more importantly, the occurrence of delayed incisional hernia may be prevented.

This case suggests that $\mathrm{CT}$ of the abdominal wall may be a useful tool for the diagnosis of even limited abdominal wound dehiscence when not accompanied by the usual signs and if the diagnosis is in doubt.

RAFII, M., ETTENGER, N., GOLIMBU, C. \& NAIDICH, D. (1983). The use of computed tomography in the evaluation of chest wall pathology. Journal of Computed Tomography, 7, 399.

RUBENSTEIN, W., AUH, Y. \& KAZAM, E. (1983) Computed Tomography of the Abdomen. Advances in Surgery, 17, 171.

STRICKLAND, B. (1984). Computed tomography of the thorax. Postgraduate Medical Journal, 60, 208.

WEBB, R. (1983). Advances in computed tomography of the thorax. Radiologic Clinics of North America, 21, 723. 